\author{
Military Technical College \\ Kobry El-Kobbah, \\ Cairo, Egypt
}

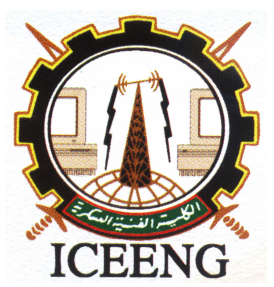

\section{$11^{\text {th }}$ International Conference on Electrical Engineering ICEENG 2018}

\title{
A New Sidelobe Cancellation Method for LFM Radars
}

By

\author{
Ahmed Azouz, Sameh Ghanem, and Abdelrahman Elbardawiny
}

\begin{abstract}
Pulse Compression technique is a vital tool commonly used in radar to increase range resolution and signal to noise ratio. Pulse compression allows achieving the performance of a shorter pulse using a longer pulse and hence gain of a large spectral bandwidth. Unwanted signals from sidelobes returns affect the detection capability of any radar. Different sidelobe reduction/cancellation techniques based on pulse compression for Linear Frequency Modulated (LFM) radars have been deployed and addressed before. In this paper, a new optimum filter for enhancing radar detection capabilities of LFM radars is introduced. The proposed filter response is compared with the windowed classical matched filter response associated with Hamming window function. The filter is implemented using Software Defined Radio (SDR). A practical test has been carried to investigate its performance. Results show superior performance of our proposed matched filter compared to that of classical versions
\end{abstract}

Index Terms- Pulse compression, LFM, Optimum filter, Range resolution, Sidelobe cancellation, SDR.

\section{INTRODUCTION}

Pulse Compression (PC) techniques are used to obtain range resolution advantage of short pulses while maintaining long range detection ability of wider transmitted pulses. It is usually implemented through modulating the transmitted waveform in either phase or frequency providing a method to further resolve targets which may have overlapping returns [1].

The costs of applying pulse compression include added transmitter and receiver complexity, and must contend with time sidelobes masking weak useful signal appearance. Sidelobe reduction/cancellation techniques have been widely used to overcome this problem and consequently enhance response of the identified or matched filter $[1,2]$.

For LFM signal, range resolution is given by: 


$$
\delta R=\frac{c \tau_{o}}{2}=\frac{c}{2 B}
$$

where, $\delta \mathrm{R}$ is the resolution in range, $\mathrm{c}$ is speed of light, to is compressed pulse duration and $B$ is the receiver bandwidth.

It is obvious that increasing receiver bandwidth yields to improvement in range resolution.

$$
P C R=\frac{\text { widthof the pulsebefore compression }}{\text { widthof the pulseafter compression }}
$$

After pulse compression and matched filtering, many unwanted lobes are generated which degrades the radar performance due to masking or hiding of small targets [3], where Peak Sidelobe Level (PSL) is given by:

$$
\operatorname{PSL}(d B)=20 \log \frac{\text { Amplitude of peak sidelobe }}{\text { Ampolitude of mainlobe }}
$$

Different modulation types for pulse compression have been widely used in radar; these may include the most famous phase-coded and LFM [4], a new manner of pulse compression for simple pulse where the desired signal waveform has a frequency characteristics selected to get low peak sidelobe levels [5], and a proposed phase coded pulse compression mechanism to explain the weather targets return is presented in [6] with performance comparison of various filters and inverse filters used to suppress the unwanted sidelobe.

In [4], Different sidelobe suppression methods of LFM waveform are introduced including comparative behavior of LFM and NLFM waveform with low pulse compression ratio. Convolutional windowing in time domain used for LFM sidelobe suppression to get lower sidelobe compression output is discussed in [7]. Analysis and demonstration of sidelobe level reduction in sonar imaging using stepped-frequency pulses is introduced in [8].

In this paper, we introduce a new optimum matched filter that completely cancel sidelobes levels in any LFM radar systems without the use of any additional weighting or windowing functions as needed in conventional ones. The rest of this paper is organized as follows: Section 2 introduces matched filter response and sidelobe level reduction by windowing. Section 3 presents the proposed optimum filter structure. This is followed by performance analysis of the suggested optimum filter response for LFM radar signal compared to traditional windowed matched filter response in Section 4. Section 5 presents real implementation and testing of the proposed optimum filter using SDR. Finally, some concluding remarks and future work proposals are given in Section 6 .

\section{Matched Filter Response}

The transmitted LFM waveform of a single amplitude modulated rectangular pulse can be described as [9]: 


$$
x(t)=A \operatorname{rect}\left(\frac{t}{T}\right) \exp \left[j\left(2 \pi f_{o} t+\pi K t^{2}\right)\right]
$$

where $A$ is the signal amplitude, $t$ is the time, $T$ is the pulse width, $f_{o}$ is the carrier frequency, $K$ is the LFM slope, while the rect ( ) function is defined as:

$$
\operatorname{rect}(x)= \begin{cases}1 & |x|<1 / 2 \\ 0 & |x|>1 / 2\end{cases}
$$

LFM slope is specified by $K= \pm B / T$, the positive sign indicates up LFM slope (up chirp) while negative sign indicates a down LFM slope (down chirp), and $B$ is the chirp bandwidth. The amplitude modulation is $A_{T}(\mathrm{t})=A \operatorname{rect}(t / T)$ and the phase modulation is represented as a quadratic function of time as [9]:

$$
\phi(t)=\pi K t^{2}
$$

Frequency modulation, defined as the immediate frequency deviation from the carrier frequency (fo) is expressed in terms of the phase by:

$$
f_{i}(t)=\frac{1}{2 \pi} \frac{d \phi(\mathrm{t})}{d t}
$$

Frequency modulation for LFM waveform is linear with slope equal to $(\alpha)$

$$
f_{i}(t)=K t= \pm\left(\frac{B}{T}\right) t, \quad|t| \leq T / 2
$$

The received signal is reflected back to the radar. The echoed signal from target can be expressed as:

$$
u(t)=A_{r} \operatorname{rect}\left(\frac{(t-\tau)}{T}\right) \exp \left[2 \pi f_{o}(t-\tau)+\pi K(t-\tau)^{2}\right]
$$

where: $A_{r}$ is an attenuated version of $\mathrm{A}, \tau$ is the two-way time of flight to the target at range $R$. Then $\tau$ can be obtain as follows:

$$
\tau=\frac{2 R}{c}
$$

where c light speed in atmosphere.

Applied matched filter response in the frequency domain is shown in Figure 1.

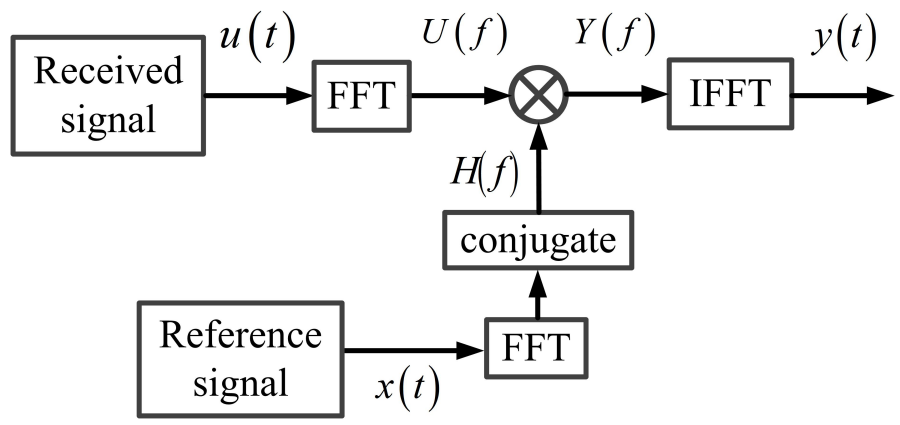

Fig. 1. Matched filter components in the frequency domain [9]

Matched filter impulse response is described by [10] 


$$
H(f)=(F F T[x(t)])^{*}=A_{H} \operatorname{rect}\left[\frac{\left(f-f_{o}\right)}{|K| T}\right] \cdot \exp \left(+j \pi \frac{\left(f-f_{o}\right)^{2}}{K}\right)
$$

where FFT is the fast Fourier transform, ${ }^{*}$ is the conjugate, and $A_{H}$ is the amplitude of matched filter impulse response.

$$
U(f)=F F T[u(t)]=A_{r}^{\prime} \operatorname{rect}\left[\frac{\left(f-f_{o}\right)}{|K| T}\right] \cdot \exp \left(-j \pi \frac{\left(f-f_{o}\right)^{2}}{K}\right) \cdot \exp (-j 2 \pi f \tau)
$$

where $A_{r}^{\prime}$ is the amplitude of matched filter impulse response.

Spectrum of the matched filter output after pulse compression is given by:

$$
Y(f)=F F T[u(t)] \cdot H(f)=A_{Y} \operatorname{rect}\left[\frac{\left(f-f_{o}\right)}{|K| T}\right] \exp (-j 2 \pi f \tau)
$$

Where $A_{Y}$ is the amplitude of spectrum of the matched filter output after pulse compression. The compressed signal in time domain is given by inverse Fourier transform (IFFT) of $Y(f)$.

$$
y(t)=\operatorname{IFF} T[Y(f)]=A_{Y}|K| T \operatorname{sinc}(K T(\mathrm{t}-\tau))
$$

A window is used after matching filtering process to eliminate the impact level of sidelobes generated at the filter output.

There are many windowing techniques used in sidelobe elimination or reduction such as Hamming, Hanning, Flattop, and Blackman windowing filters. It has been shown that Hamming windowing has a better performance among other mentioned filter techniques w.r.t resolution and peak of sidelobe level [11].

\section{Proposed Optimum Filter Structure}

Our proposed filter is designed to completely cancel any sidelobe levels presented after matching filtering of LFM signals as shown in Figure 2.

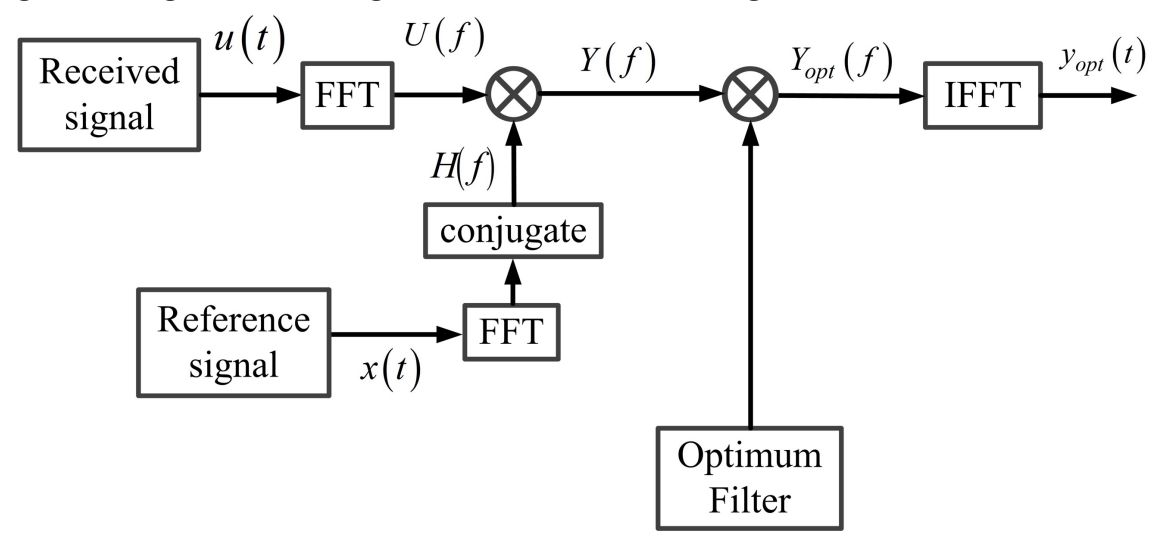

Fig. 2. Optimum filter for side lobe cancellation 
The general form in digital form of a single-pulse LFM signal in (10) can be expressed as:

$$
\mathrm{s}(\mathrm{n})=A \mathrm{e}^{\mathrm{J}\left(\mathrm{w}_{\mathrm{o}} \mathrm{n}+\mathrm{k} \pi \mathrm{n}^{2}\right)} \quad 0 \leq \mathrm{n} \leq \mathrm{N}-1
$$

Where $\mathrm{A}$ is the amplitude, fs is the sampling frequency, $\omega \mathrm{O}$ is the angular frequency, $\mathrm{k}=$ $\mathrm{B} /\left(\mathrm{Nf}_{\mathrm{s}}\right)$ which is LFM coefficient, and $\mathrm{N}$ is number of samples.

We have two scenarios for optimum filter construction according to the value of integer $\mathrm{N}$, odd or even. Matched filter autocorrelation function is expressed by:

$$
\mathrm{x}_{\mathrm{N}}(\mathrm{l})=\sum_{\mathrm{n}=-\infty}^{\infty} \mathrm{s}_{\mathrm{N}}^{*}(\mathrm{n}) \mathrm{s}_{\mathrm{N}}(\mathrm{n}+\mathrm{l}) \quad 0 \leq \mathrm{l} \leq \mathrm{N}-1
$$

where the asterisk $\left({ }^{*}\right)$ indicates complex conjugate.

\section{Odd number samples}

Discrete Fourier Transform (DFT) of autocorrelated signal for odd number of samples (let $\mathrm{N}=5$ ) can be expressed as:

$$
\begin{aligned}
X_{5}(\omega)= & \sum_{l=0}^{M-1} x_{5}(l) e^{-j \frac{2 \pi l \omega}{M}} \quad \text { where } M=9 \\
= & e^{-j 4\left(k \pi+2 \omega+\omega_{o}\right)}\left\{e^{j 4(k \pi+2 \omega)}+5 e^{j 4\left(k \pi+\omega+\omega_{o}\right)}+e^{j 2\left(3 \omega+\omega_{o}\right)}+e^{j 2\left(2 k \pi+3 \omega+\omega_{o}\right)}+\right. \\
& e^{j 2\left(4 k \pi+3 \omega+\omega_{o}\right)}+e^{j\left(k \pi+7 \omega+\omega_{o}\right)}+e^{j\left(7 k \pi+7 \omega+\omega_{o}\right)}+e^{j 4\left(k \pi+2 \omega_{o}\right)}+e^{j 2\left(\omega+3 \omega_{o}\right)}+ \\
& e^{j 2\left(2 k \pi+\omega+3 \omega_{o}\right)}+e^{j 2\left(4 k \pi+\omega+3 \omega_{o}\right)}+e^{j\left(k \pi+5 \omega+3 \omega_{o}\right)}+e^{j\left(3 k \pi+5 \omega+3 \omega_{o}\right)}+ \\
& e^{j\left(5 k \pi+5 \omega+3 \omega_{o}\right)}+e^{j\left(7 k \pi+5 \omega+3 \omega_{o}\right)}+e^{j\left(k \pi+3 \omega+5 \omega_{o}\right)}+e^{j\left(3 k \pi+3 \omega+5 \omega_{o}\right)}+ \\
& \left.e^{j\left(5 k \pi+3 \omega+5 \omega_{o}\right)}+e^{j\left(7 k \pi+3 \omega+5 \omega_{o}\right)}+e^{j\left(k \pi+\omega+7 \omega_{o}\right)}+e^{j\left(7 k \pi+\omega+7 \omega_{o}\right)}\right\}
\end{aligned}
$$

Output response in time domain, $\mathrm{y}(\mathrm{n})$, for $\mathrm{N}=5$ samples is set as:

$$
y_{5}(l)=\{0,0,0,0,5,0,0,0,0\}
$$

Spectrum of the output response of (16) can be rewritten as:

$$
Y_{5}(\omega)=5 e^{-j 4 \omega}
$$

The proposed optimum filter transfer function for 5 samples and its simplified form in frequency can be fulfilled as in (18) and (19) respectively.

$$
H_{5}(\omega)=\frac{Y_{5}(\omega)}{X_{5}(\omega)}=\frac{5}{\left\{\begin{array}{c}
2 \cos \left(4\left(\omega-\omega_{o}\right)\right)+2 \cos \left(3\left(k \pi+\omega-\omega_{o}\right)\right)+ \\
2 \cos \left(2\left(2 k \pi-\omega+\omega_{o}\right)\right)+2 \cos \left(2\left(\omega-\omega_{o}\right)\right)+2 \cos \left(k \pi+\omega-\omega_{o}\right)+ \\
2 \cos \left(k \pi-\omega+\omega_{o}\right)+2 \cos \left(3 k \pi+\omega-\omega_{o}\right)+2 \cos \left(3 k \pi-\omega+\omega_{o}\right)
\end{array}\right\}}
$$

Simplified to: 


$$
H_{5}(\omega)=\frac{5}{\left\{\begin{array}{c}
5+4[\cos (k \pi)+\cos (3 k \pi)] \cos \left(\omega-\omega_{o}\right)+ \\
2[1+2 \cos (4 k \pi)] \cos \left(2\left(\omega-\omega_{o}\right)\right)+ \\
4 \cos (3 k \pi) \cos \left(3\left(\omega-\omega_{o}\right)\right)
\end{array}\right\}}
$$

Similarly, the proposed optimum filter transfer function for 7 samples can be simplified as:

$$
H_{7}(\omega)=\frac{7}{\left\{\begin{array}{c}
7+4[\cos (k \pi)+\cos (3 k \pi)+\cos (5 k \pi)] \cos \left(\omega-\omega_{o}\right)+2 \cos \left(6\left(\omega-\omega_{o}\right)\right) \\
2[1+2 \cos (4 k \pi)+2 \cos (8 k \pi)] \cos \left(2\left(\omega-\omega_{o}\right)\right)+ \\
4[\cos (3 k \pi)+\cos (9 k \pi)] \cos \left(3\left(\omega-\omega_{o}\right)\right)+ \\
2[1+2 \cos (8 k \pi)] \cos \left(4\left(\omega-\omega_{o}\right)\right)+4 \cos (5 k \pi) \cos \left(5\left(\omega-\omega_{o}\right)\right)
\end{array}\right\}}
$$

Hence, the proposed optimum filter transfer function general form for $(\mathrm{N})$ odd samples is written as:

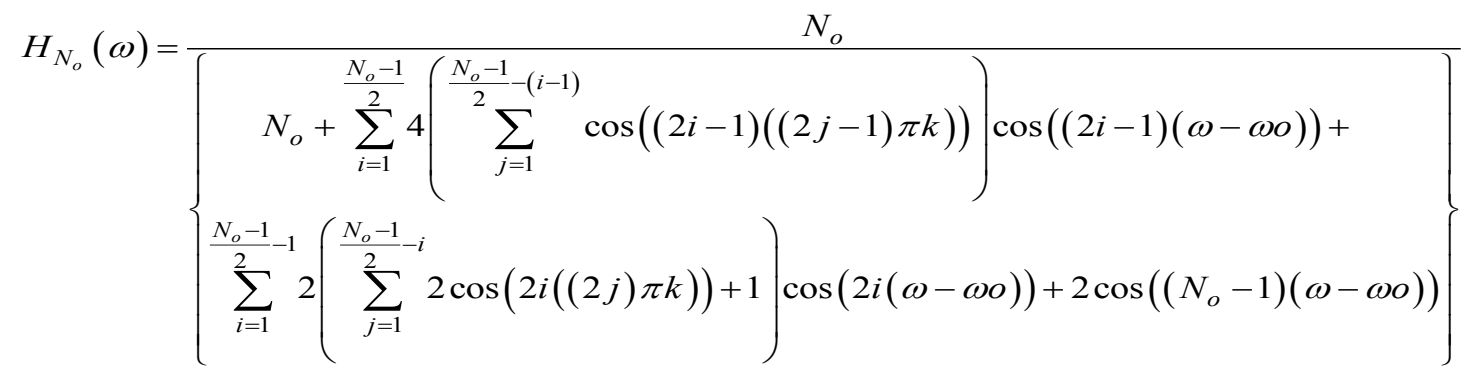

where $N_{0}$ is number of sample in reference LFM and it is odd number.

\section{Even number samples}

The proposed optimum filter transfer function for even number of samples $(\mathrm{N}=6)$ can be simplified as:

$$
H_{6}(\omega)=\frac{6}{\left\{\begin{array}{l}
6+2[1+2 \cos (2 k \pi)+2 \cos (4 k \pi)] \cos \left(k \pi+\omega-\omega_{o}\right)+ \\
4[\cos (2 k \pi)+\cos (6 k \pi)] \cos \left(2\left(k \pi+\omega-\omega_{o}\right)\right)+ \\
2[1+2 \cos (6 k \pi)] \cos \left(3\left(k \pi+\omega-\omega_{o}\right)\right)+ \\
4 \cos (4 k \pi) \cos \left(4\left(k \pi+\omega-\omega_{o}\right)\right)+2 \cos \left(5\left(k \pi+\omega-\omega_{o}\right)\right)
\end{array}\right\}}
$$

And for 8 samples, the transfer function can be obtained by:

$$
\begin{aligned}
& H_{8}(\omega)=\frac{8}{\left(8+2[1+2 \cos (2 k \pi)+2 \cos (4 k \pi)+2 \cos (6 k \pi)] \cos \left(k \pi+\omega-\omega_{o}\right)+\right]} \\
& 4[\cos (2 k \pi)+\cos (6 k \pi)+\cos (10 k \pi)] \cos \left(2\left(k \pi+\omega-\omega_{o}\right)\right)+ \\
& 2[1+2 \cos (6 k \pi)+2 \cos (12 k \pi)] \cos \left(3\left(k \pi+\omega-\omega_{o}\right)\right)+ \\
& 4[\cos (4 k \pi)+\cos (12 k \pi)] \cos \left(4\left(k \pi+\omega-\omega_{o}\right)\right)+ \\
& 2[1+2 \cos (10 k \pi)] \cos \left(5\left(k \pi+\omega-\omega_{o}\right)\right)+ \\
& 4 \cos (6 k \pi) \cos \left(6\left(k \pi+\omega-\omega_{o}\right)\right)+2 \cos \left(7\left(k \pi+\omega-\omega_{o}\right)\right)
\end{aligned}
$$


Hence, the transfer function general form for even samples $(\mathrm{N})$ can be expressed from (22) and (23) as:

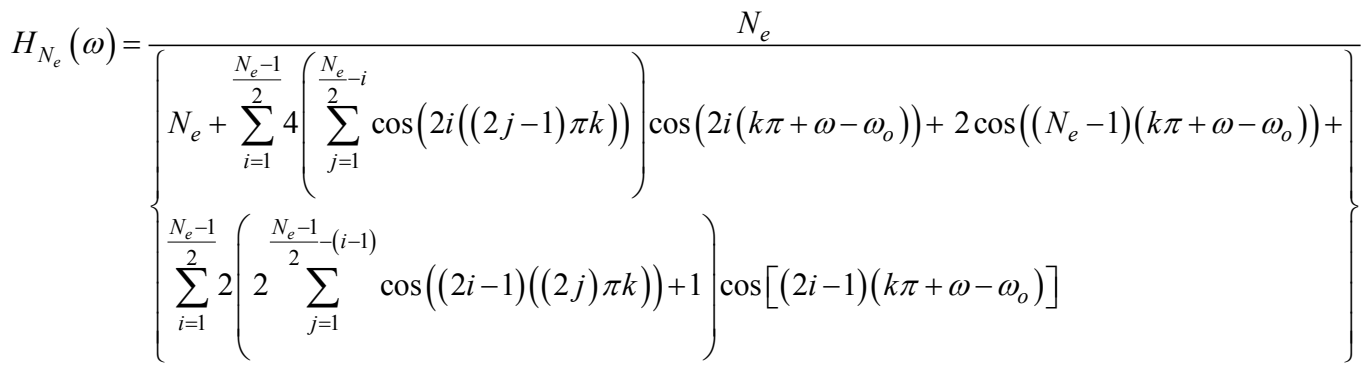

$$
\begin{aligned}
& \text { where } N_{e} \text { is number of sample in reference LFM and it is even number. }
\end{aligned}
$$

\section{Proposed Filter Performance Analysis}

Performance analysis and evaluation of the proposed optimum filter is carried out in MATLAB. Base band signal of 1024 samples, $120 \mathrm{MHz}$ sampling frequency, and $100 \mathrm{MHz}$ bandwidth is generated. Simulation is carried using White Gaussian thermal noise with zero mean and unity variance environment. Detailed comparison of our proposed filter output sidelobe reduction level with that of Hamming windowing filter is presented after. Also, detection probability and range resolution were taken into consideration as in Figure 3.

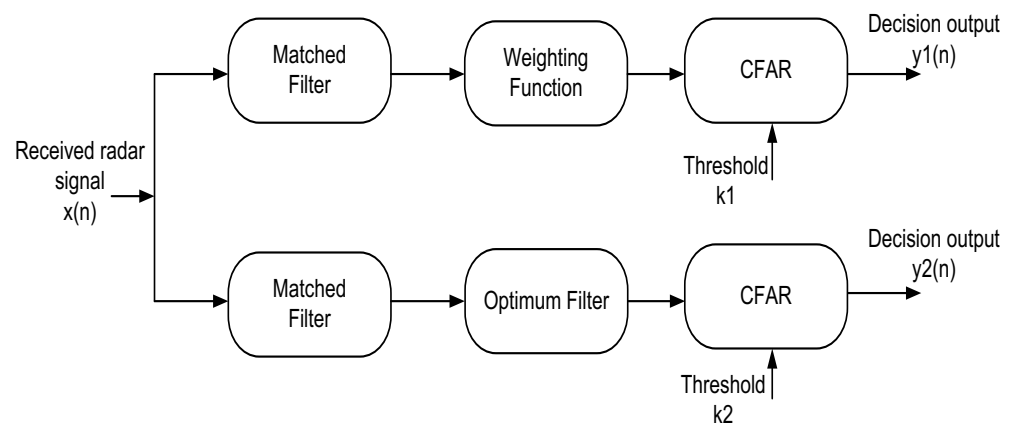

Fig. 3. Performance evaluation setup of the suggested optimum filter compared to the weighting filter

\section{Detection evaluation}

Detection performance is evaluated for both of proposed filter and conventional matched filter with Hamming window through Receiver Operating Characteristics (ROC) under certain false alarm probability $(\mathrm{Pfa})$. Detection probability $(\mathrm{Pd})$ is calculated using the Cell Average Constant False Alarm Rate (CA-CFAR) processing for both filters outputs.

Figure 4 shows the two filters responses for two separate targets at different SNRs (10dB and $5 \mathrm{~dB}$, resp.) and Pfa of 10-6. 


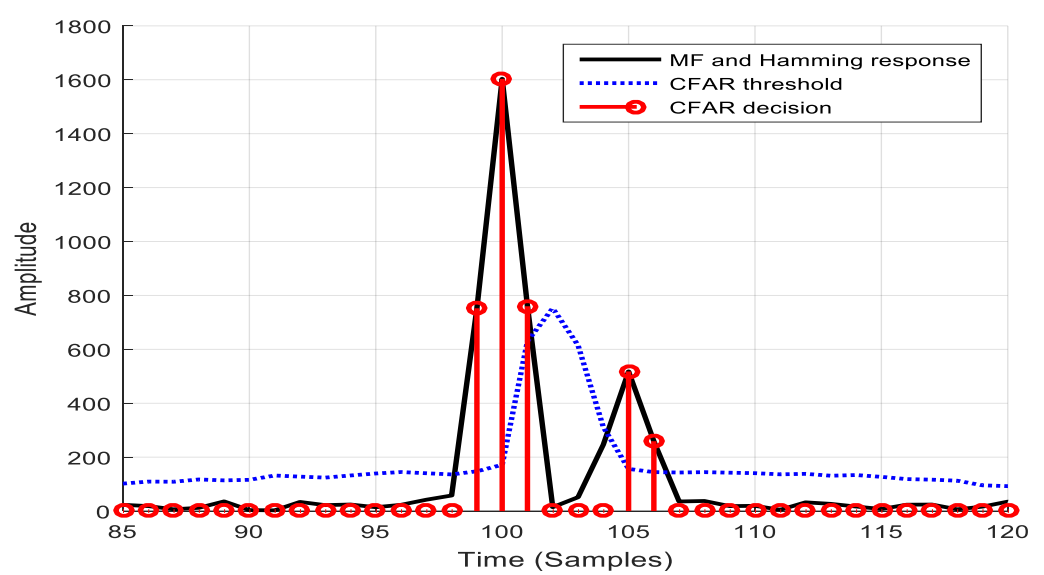

(a)

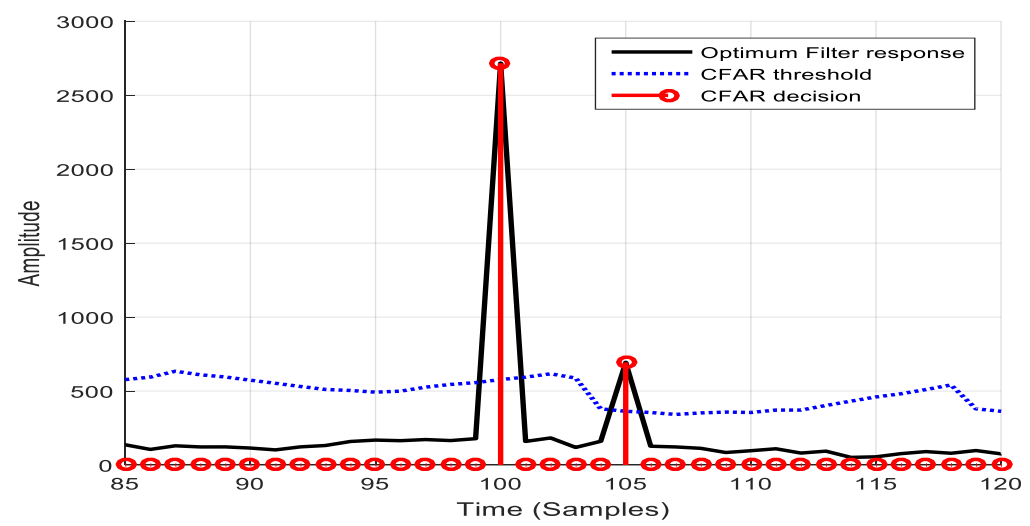

(b)

Figure 4. CFAR detection for two targets at $P f a=10-6$ and $(S N R=10 \mathrm{~dB}$ and $5 \mathrm{~dB})(\mathrm{a})$ Hamming windowing filter, (b) Proposed optimum filter

As shown above, matched filter detection with Hamming window has a bad response compared with that of the proposed filter due to high sidelobe levels introduced at its output.

There are many false targets detected due to low SNR as presented in Figure 4(a) while as shown in figure 4(b), the predefined two known targets were only detected indicating better detection capabilities of our proposed filter.

ROC curves of both proposed and conventional matched with/without Hamming window filters at Pfa of 10-6 are shown in Figure 5. 


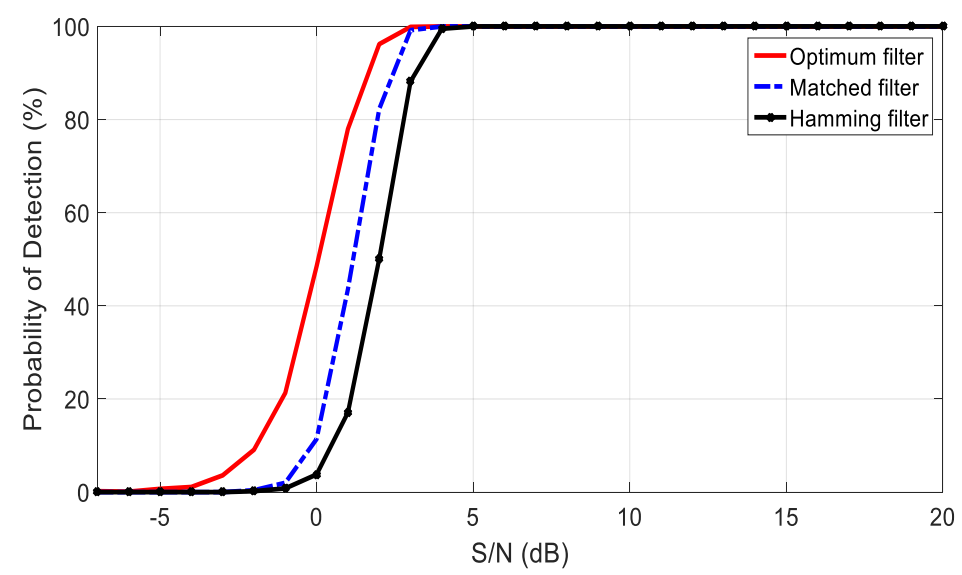

Figure 5. ROC for proposed and conventional matched with/without Hamming window filters at $\mathrm{Pfa}=10-6$

Clearly, optimum filter detection performance outperforms that of the conventional one by approximately $2.5 \mathrm{~dB}$ and outperforms the conventional one with Hamming window by approximately $3 \mathrm{~dB}$.

\section{Resolution performance}

Range resolution of the proposed optimum filter for LFM radar signal is evaluated and compared with that of the conventional matched with/without Hamming window filtering. Simulation is performed for two closely spaced targets at two range cells number 100 and 102 respectively, which practically are separated by one range cell. CFAR detection outputs of these targets in range domain at different SNRs (10 dB and $15 \mathrm{~dB})$ and Pfa of 10-6 are calculated. Figure 6(a) represents CFAR output detection for conventional matched filter without windowing showing difficulty in discriminating the two targets.

Figure 6(b) shows CFAR output for conventional matched Filtering with Hamming window. Range resolution is worse than that of the conventional one without windowing. This is obvious because of windowing effect which reduces sidelobe levels on behalf of resolution.

CFAR detection of the proposed filter is shown in Figure 6(c), the two targets appear clearly indicating better discrimination and enhanced range resolution. 


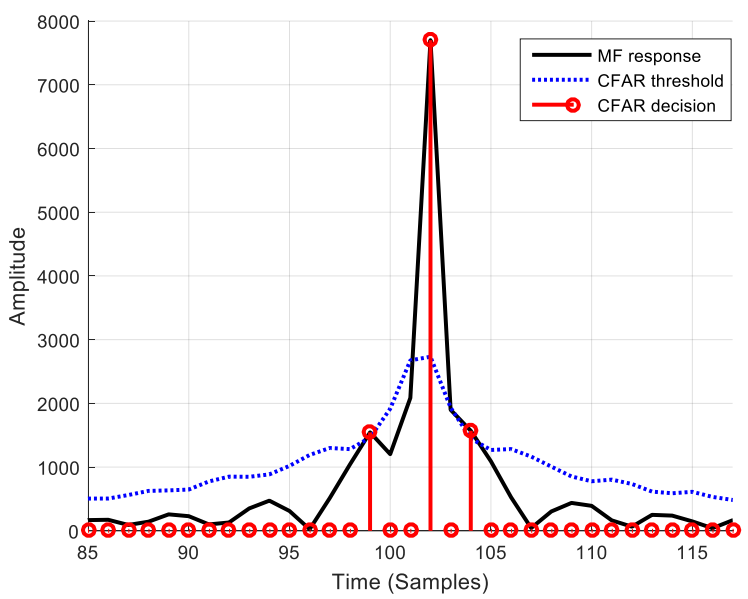

(a)

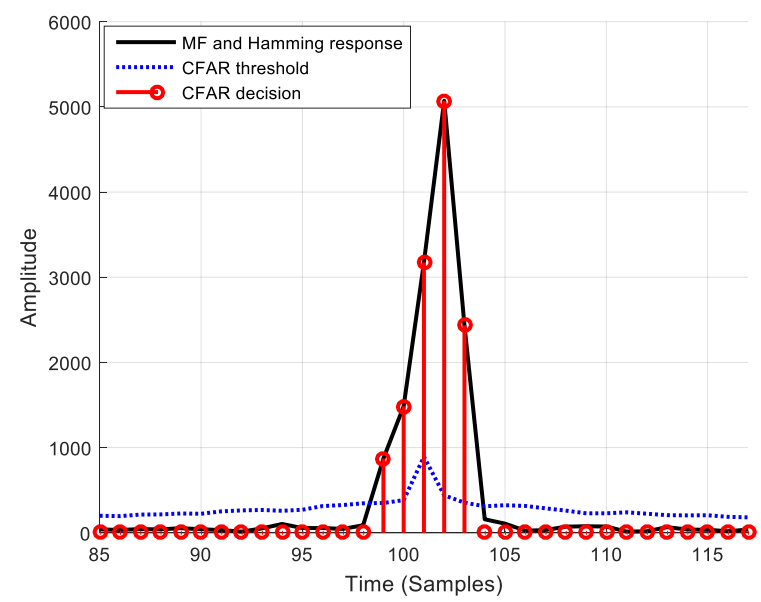

(b)

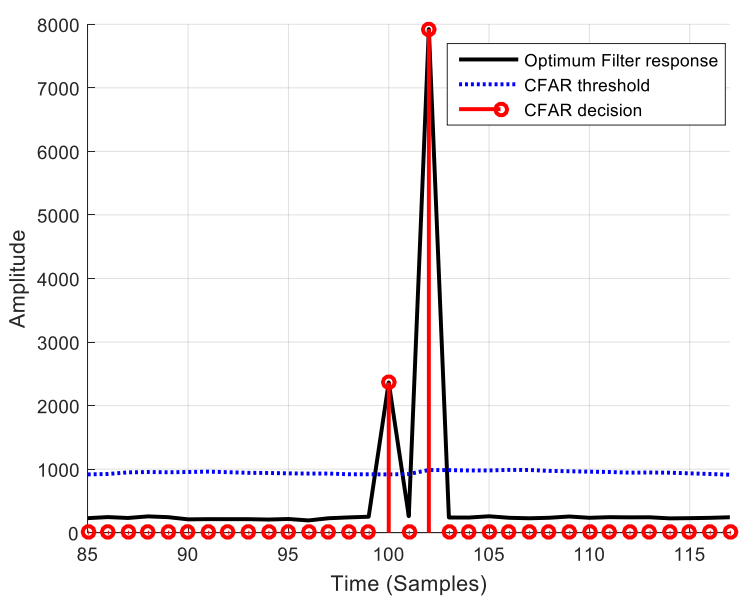

(c)

Fig. 6. CFAR output for two closely spaced targets at $P f a=10-6$ and $(S N R=10 \mathrm{~dB}$ and 15dB): (a) Conventional matched filter, (b) Conventional matched filter with Hamming window, and (c) Proposed Optimum filter 


\section{Peak sidelobe reduction}

Compared to both matched and Hamming windowing filters; as $\mathrm{S} / \mathrm{N}$ increases, sidelobes levels at the proposed filter output decrease. PSLR ratio is linearly decreasing with increasing of $\mathrm{S} / \mathrm{N}$ above $10 \mathrm{~dB}$, where we can almost find any sidelobes as shown in Fig.7. We can say that; PSLR ratio of the proposed optimum filter outperforms that of other filters due to the absence of any sidelobes at its output.

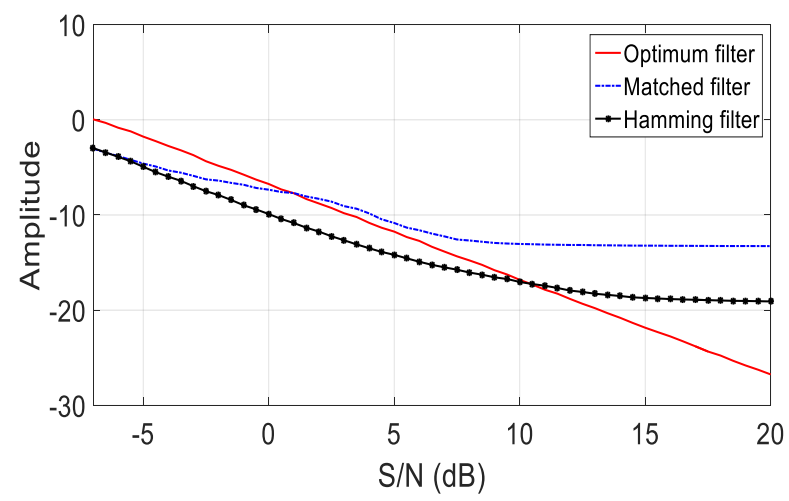

Fig. 7. Effect of increasing SNR on the PSLR using optimum filter compared to conventional matched filter with/without Hamming window

\section{Implementation of the Proposed Optimum Filter using SDR}

The proposed optimum filter is implemented using PXI (a rugged PC-based platform for measurement and automation systems by National Instruments) to achieve the filter performance in actual time applications. Vector signal generator and vector signal analyzer are combined together in Vector Signal Transceiver (VST) with signal processing and control established on real-time FPGA such as NI PXIe-5644R. The suggested filter is implemented using PXI with $120 \mathrm{MHz}$ sampling rate, $2.4 \mathrm{GHz}$ carrier frequency, dual-channel $1600 \mathrm{MHz}$ DDR3 memory and $100 \mathrm{MHz}$ bandwidth [12].

\section{Experiment Setup}

This experiment includes two antennas working properly in S-Band $(2.4 \mathrm{GHz})$ for transmitting and receiving data, NI PXIe-5644R VST as SDR platform, A corner reflector and a flat plate are used as first and second targets respectively. NI LabVIEW FPGA Model based software is used to perform the signal generation and processing in base band. The signal processor shown in Figure 3 is built at the receiver part using the proposed optimum filter in frequency domain. The experiment setup and its implementation setting are presented in Figure 8. 


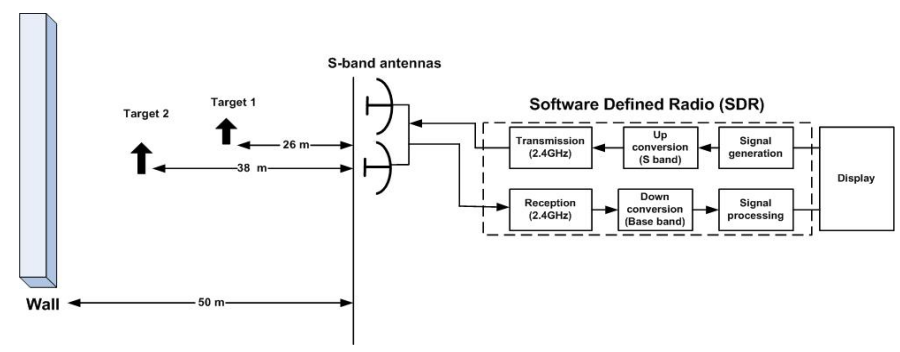

(a)

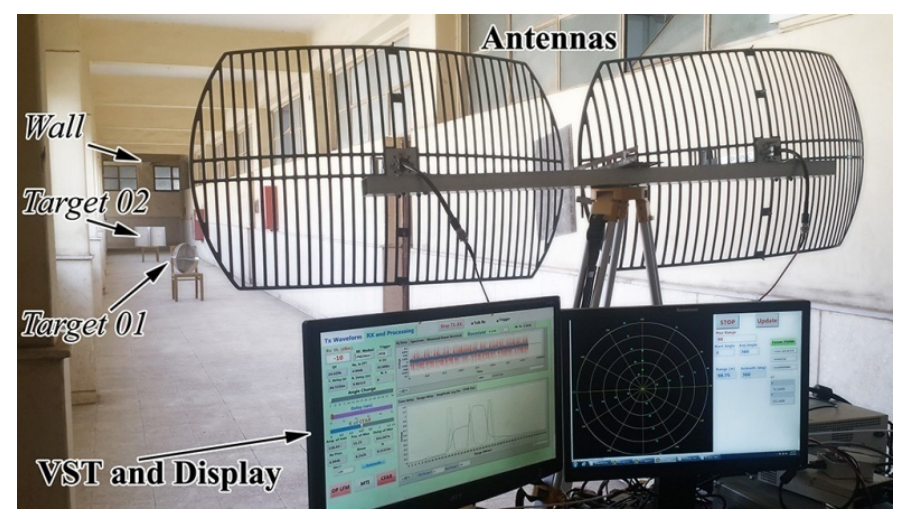

(b)

Fi. 8. Experiment setup (a) Block diagram, (b) Real photo

\section{Experiment results}

Signal Generation: LFM transmitted signal $(2.4 \mathrm{GHz}$ carrier frequency, $50 \mathrm{MHz}$ bandwidth, Pulse Repetition Interval (PRI) of $21.6667 \mu \mathrm{sec}$ with $76.9 \%$ duty cycle, and $2.6 \mathrm{~K}$ samples per PRI), I \& Q channels signals in base band with $15 \mathrm{dBm}$ transmitted signal power are generated as shown in Figure 9.

Received Signal: Average received power $\approx-21.43 \mathrm{dBm}$, in the band from 2.37 to 2.45 $\mathrm{GHz}$.

Matched filter processing: Figure 10(a), solid blue line, represents the matched filter output with high sidelobe levels, while figure $10(b)$ is its log representation, also in solid blue line.

Proposed optimum filter processing: Figure 10(a), dashed red line, represents the proposed filter output where high sidelobe levels have been removed, while figure 10(b) is its log representation, also in dashed red line. 


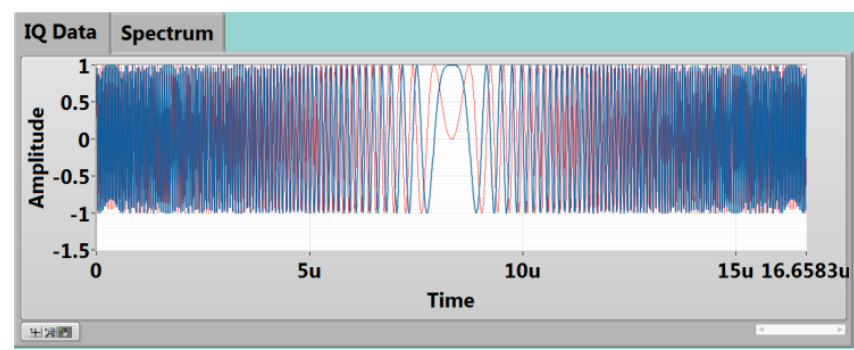

(a)

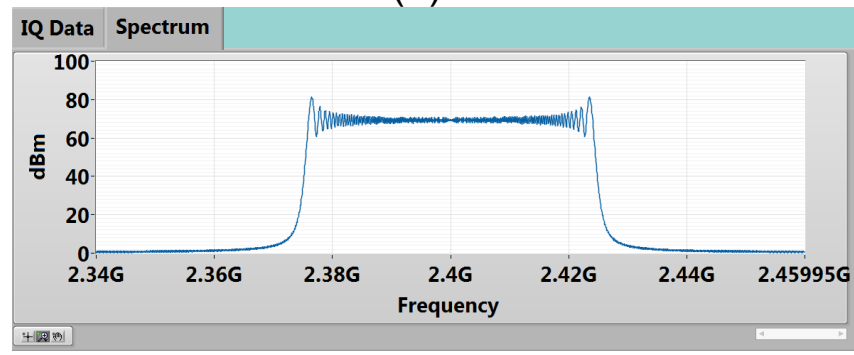

(b)

Fig. 9. Transmitted radar signal: (a) I and Q channels, (b) Radar signal spectrum

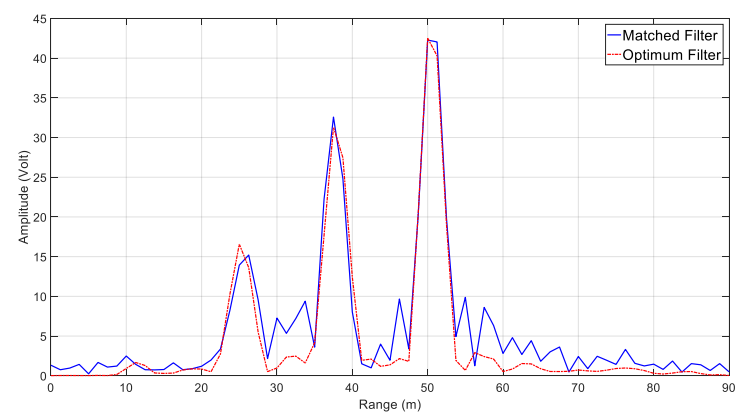

(a)

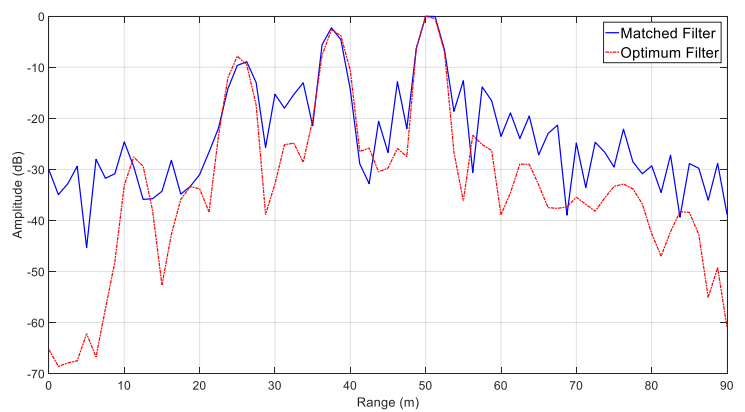

(b)

Fig. 10. Output responses of matched and proposed filters without CFAR

Target Detection: CFAR processor is used for target discovery with both the conventional matched filter and the proposed optimum filter. Figure 11 represents the detection of two targets with different cross sections placed at ranges of 25 and 37 meter respectively. In Figure 11(a), conventional matched filter detection of the two targets is shown beside a strong return detection from the back wall placed at 51 meter apart. The CFAR processor 
passed the detected targets to the radar display front panel which is adjusted to 90 meters as maximum range. CFAR threshold, based on the input from the matched filter, is calculated and is shown by dashed red line. Figure 11(b) represents the output of our proposed optimum filter for the same scenario of conventional one before. Although the CFAR successfully detected the two targets and the wall in both situations, it failed in the case of conventional matched filter to avoid high sidelobe levels from the wall. It considered the last sidelobe as target (false one). While in our proposed filter, these unwanted sidelobes are completely removed, and there is no chance for any false detection.

\section{Experiment Analysis}

Quantitative comparison of Peak to Sidelobe Ratio (PSLR) and resolution, of the proposed optimum filter and the conventional matched filter with/without Hamming windowing at $10 \mathrm{~dB}$ SNR and $120 \mathrm{MHz}$ sampling frequency, has been calculated and presented in table 1. It is found that, the proposed optimum filter has better performance than that of the conventional matched one with/without the use of Hamming windowing.

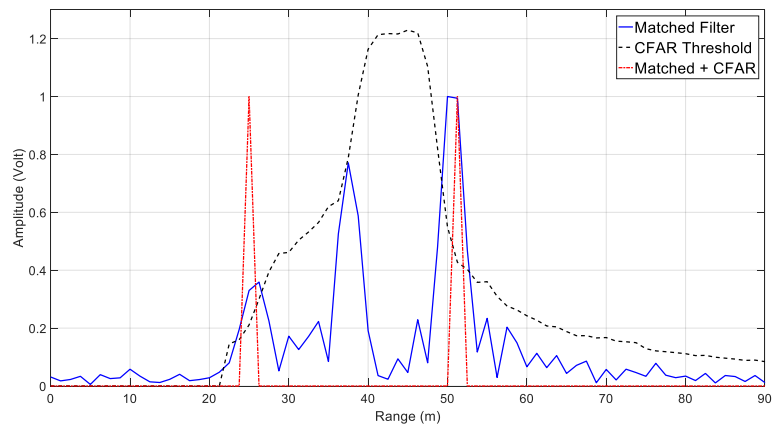

(a)

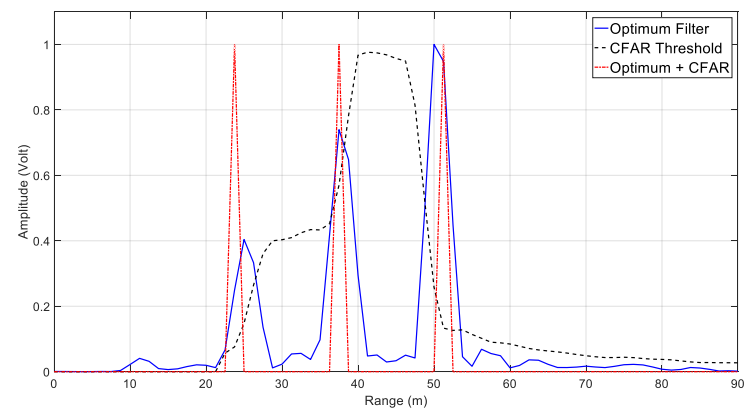

(b)

Fig. 11. Target detection with CFAR processing of: (a) Conventional matched filter, (b) Proposed optimum filter 


\section{Conclusion}

In this paper, a new filter design, completely cancels sidelobe levels in LFM radar signals, has been introduced. Interpretation of the proposed optimum filter compared to that of the conventional matched filter associated with Hamming windowing has been evaluated and analyzed through ROC curves. The proposed optimum filter has a good detection performance above $10 \mathrm{~dB}$ of SNR. It outperforms the conventional matched one associated with Hamming windowing in sidelobe reduction or cancelation. It also has a better range resolution compared to that of both conventional matched filter with/without Hamming windowing. Implementation of the proposed optimum filter using SDR (mainly PXI and programmed using LabVIEW) is introduced, and its performance has been evaluated practically to verify both theoretical and simulation results. Practical experiment assures the simulation results achieved and shows the superior removal of sidelobe levels of LFM signal with better resolution capability introduced by our proposed optimum filter compared to that of the conventional matched filter alone or with the use of Hamming windowing.

\section{References}

[1] M. I. Skolnik, Introduction to Radar Systems. 3rd ed., McGraw-Hill, New York, 2002.

[2] M.H. Ackroyd, and F. Ghani, "Optimum Mismatched Filters for Sidelobe Suppression," IEEE Trans. on Aerospace and Electronic Systems, Vol. AES-9, Issue 2, pp. 214-218, March 1973.

[3] C. D. Rawat and A. D. Sarate, "High Resolution Low Power Radar Pulse Compression Techniques," International Journal of Advanced Research in Electrical, Electronics and Instrumentation Engineering (IJAREEIE), Vol. 3, Issue 4, pp. 89288935, 2014.

[4] L. R. Varshney and D. Thomas,"Sidelobe Reduction for Matched Filter Range Processing," in Proceedings of the 2003 IEEE Radar Confererence, pp. 446-451, May 2003.

[5] M. Shinriki et al, "Pulse Compression for a simple pulse," IEEE Trans. on Aerospace and Electronic Systems, Vol. 44, Issue 4, pp. 1623-1629, October 2008.

[6] A. S. Mudukutore et al, "Pulse compression for weather radars," IEEE Trans. on Geoscience and Remote Sensing, Vol. 36, Issue 1, pp. 125-142, January 1998.

[7] A. K. Sahoo and G. Panda, "Sidelobe reduction Of LFM signal using convolutional windows," International Journal of Signal and Imaging Systems Engineering, Vol. 5, Issue 4, pp. 253-259, 2012.

[8] X. Liu et al, "Low Sidelobe Range Profile Synthesis for Sonar Imaging using SteppedFrequency Pulses," IEEE Geoscience and Remote Sensing Letters, Vol. 14, Issue 2, pp. 218-221, February 2017. 
[9] B. R. Mahafza, Radar Systems Analysis and Design using Matlab, 3rd ed., RCR press, Alabama, USA, 2013.

[10] N. Levanon and E. Mozeson, Radar signals, John Wiley \& Sons Inc., Hoboken, NewJersey, USA, 2004.

[11]M. Archana and M. Gnana, "Low power LFM Pulse Compression Radar with Sidelobe Suppression," International Journal of Advanced Research in Electrical, Electronics and Instrumentation Engineering (IJAREEIE), Vol. 3, Issue 7, pp. 10627-10679, July 2014.

[12]National Instruments user manuals. Available: http://www.ni.com/pxi/, National Instruments (NI), 2015. 